\title{
Process Capability Optimization in Multistage Manufacturing Processes Based on the Cost-Utility Ratio
}

\author{
Ning Wang*
}

Business school, Zhengzhou University, Zhengzhou 450001, P.R. China

\begin{abstract}
To solve the problem that process capability optimization in multistage manufacturing processes has not been studied so much. Firstly, a multistage manufacturing process quality variation model is built to analyze the impaction of each stage quality on process capability. Then, a multistage manufacturing process capability analysis method with "Costutility ratio" is proposed to prioritize process capability optimization efforts. At last, a two stage matching process is taken as an example to demonstrate it. The results show that this method ascertains the priorities and process capability optimization degree well.
\end{abstract}

Keywords: Multistage manufacturing process, quality improvement, process capability optimization, cost-utility ratio.

\section{INTRODUCTION}

Multistage manufacturing process capability refers to the actual processing ability of multistage manufacturing process under steady state making products to meet the design specification, which is usually affected by the specification limits, the process mean and process variance. Among them, the specification limit is assigned by the "customers", and the process mean and process variance affected by the manufacturing process. If process average is not deviated, then process capability can be improved by decreasing the process variance namely reducing the quality variation. However, as the production has become increasingly complex, the manufacturing process often involves more sub processes, in which the influence of the correlation exist, and the impact of the quality variation reduction on process capability is also more complex.

In recent years, Scholars at home and abroad have researched the subject that multistage manufacturing process capability optimization widely. Professor Zhang Gongxu presented two kinds of process capability index which is used to judge and evaluate the production process. Pearn, Chang and some scholars put forward the method of process capability analysis on the process with Multi-strip production line. Ding studied on the process capability sensitivity of the multistage manufacturing process. Liu Daoyu and Jiang Pingyu proposed a multistage process capability evaluation method based on the process variation trajectory chart to solve the problem that the single process and single quality characteristic evaluation method cannot be directly used in multistage process capability analysis. Linn, studied a multistage process capability analysis algorithm, and which is used to analyze the multistage process improvement effect. Sun Jing proposed a process capability analysis method with regard to the relevant data. Huang Wenzhen and Kong Zhenyu proposed sensitivity analysis method based on the qualification rate to evaluate the multistage assembly process capability. Fang Zhu and Xiongfei, Huang used the modified process capability indices to calculate the overall process capacity of complex product manufacturing processing, and further determined the improvement direction and scope of single process parameters based on the sensitivity analysis.

In conclusion, the current multistage manufacturing process optimization mainly through the sampling data of existing process to make "afterwards" evaluation, which is to use the experimental design method to reduce the sensitivity of the product performance to process parameter variation. The ultimate goal of these methods is to achieve the most optimal process capability. However, there is no way to effectively evaluate the direct influence of each sub processes quality variation on multistage manufacturing process capability. In addition, existing research only focuses on process variation reduction, but ignore the resource consumption with it, which would tend to cause excessive process control and ignore the fluctuations that tend to cause and form quality waste. Therefore, in this article, based on the Law less's multistage manufacturing process analysis model, the corresponding relationship between each sub process quality variation with each sub process capability, sub process transfer coefficient and total process capability is built, and the influence of each sub processes quality variation decrease and different transfer coefficient on the process capability. Then according to the "difficulty" and "cost-utility ratio" of variation reduction evaluating the quality improvement effect, so as to provide the basis for process optimization.

\section{RESEARCH ASSUMPTIONS AND VARIABLE DEFINITIONS}

Multistage manufacturing process capability optimization is mainly to identify each sub process capability in multistage manufacturing process, analyze the influence of each 
sub processes quality variation on the total process capability, then, determine the priority of each sub processes quality variation reduction in process capability optimization and confirm the degree and number in need of improvement. Therefore, in order to facilitate analysis, the following notations are used in presenting the process capability analysis procedures.

\subsection{Research Premise and Assumptions}

The multistage manufacturing process capability optimization in this paper is created under the following premise assumptions:

Premise 1: Multistage manufacturing process is stable or statistical controlled, and the process output obeys normal distribution;

Premise 2: The total process and sub-processes have clearly output characteristic;

Premise 3: The process samples extracting only affected by random factors.

Assumptions 1: To facilitate discuss $C_{\text {pmokj }}$, two stage process is chosen to illustrate multistage manufacturing process capability optimization;

Assumptions2: The random error in the two stage is $w_{X_{1}}$ and $w_{X_{2}}$, and both obey the standard normal distribution: $w_{X_{1}} \sim N(0,1), w_{X_{2}} \sim N(0,1)$;

Assumptions 3: The mean of multistage manufacturing process have deviation, the mean deviate from specification center 1 unit;

Assumptions 4: The specification ranges of both processes are $[-4,4]$, the target and specification centre are coincide, namely $T=m=0$;

Assumptions5: In the first process, $\sigma_{s 1}=\sigma_{o 1} . \sigma_{s 1}$ reflects the specific variation, which remove the quality variation affection transmitted from previous process.

\subsection{Variable Definitions}

The following notations are used in presenting the process capability optimization procedures.

$\sigma_{s i}^{2}:$ Specific variation in the process;

$\sigma_{o i}^{2}$ : Overall variation in the ith process, which is affected by the current and previous process;

$\sigma_{s i j}^{2}:$ Specific variation in the $i t h$ ith process $j$ th equipment;

$\sigma_{o i j}^{2}$ : Overall variation in the $i t h$ process $j t h$ equipment, which is affected by the current and previous process;

$\beta_{i}$ : The process transfer coefficient from stage $i-1$ to stage $i$;

$\mu_{i j}$ : Overall mean in process which is affected by the current and previous processes;

USL, LSL: The process upper and lower specification limits;
$C_{p k s i j}:$ Specific process capability in the $i t h$ process $j t h$ equipment,

$$
C_{p k s i}=\frac{\min \left(\mu_{i}-L S L, U S L-\mu_{i}\right)}{3 \sigma_{s i}}
$$

$C_{p k o i j}$ : Overall process capability in the process equipment,

$C_{p o s i}=\frac{\min \left(\mu_{i}-L S L, U S L-\mu_{i}\right)}{3 \sigma_{o i}}$

$C_{p m s i j}:$ Specific Taguchi process capability in the process equipment,

$$
C_{p m s i}=\frac{U S L-L S L}{6 \sqrt{\sigma_{s i}^{2}+\left(\mu_{i}-T\right)^{2}}}
$$

$C_{\text {pmoij }}$ : Overall Taguchi process capability in the process equipment,

$$
C_{p m o i}=\frac{U S L-L S L}{6 \sqrt{\sigma_{o i}^{2}+\left(\mu_{i}-T\right)^{2}}}
$$

$C_{p k o k j}$ : Overall process capability in the last $k t h$ process equipment.

$C_{\text {pmokj }}$ : Overall Taguchi process capability in the last process equipment,

$I C_{p k s i j}$ and $I C_{p m s i j}$ : Numerical increment of $C_{p k s i j}$ and $C_{p m s i j}$ after the variation reduction in the process equipment.

$I C_{p k o k j}$ and $I C_{p m o k j}$ : Numerical increment of $C_{p k o i j}$ and $C_{\text {pmoij }}$ after the variation reduction in the process equipment.

$F_{d i j}$ : Degree of difficulty to reduce variation in the process equipment. The degree of difficulty, $F_{d i j}$ is a normalized value among all stages. It is determined by the resource consumption on reducing the variation in each stage. The resource includes time, manpower, materials, and other overheads. The degree of difficulty for the stage $l$ with least resource consumption, is set to $1, F_{d l}=1$. The degrees of difficulty for the rest stages, $F_{d i j}$ where $i \neq l$, are determined by the ratio of resource consumptions of process $j t h$ equipment, and the stage $l$.

ER: Effort ratio which is the ratio of effort spent to increase the $C_{p k s i j}$ or $C_{p m s i j}$ with respect to the increment of the $C_{p k s i j}$ or $C_{p m s i j}$,

$$
E R=\frac{I C_{p k o k j}}{I C_{p k s i j} \times F_{d i j}}
$$




\section{MODELING ANALYSIS OF MULTISTAGE MANUFACTURING PROCESS}

Multistage manufacturing process capability analysis is based on multistage process variation propagation model. A multistage manufacturing process variation propagation model will be built in this section referring to the AR (1) model proposed by Lawless (1999).

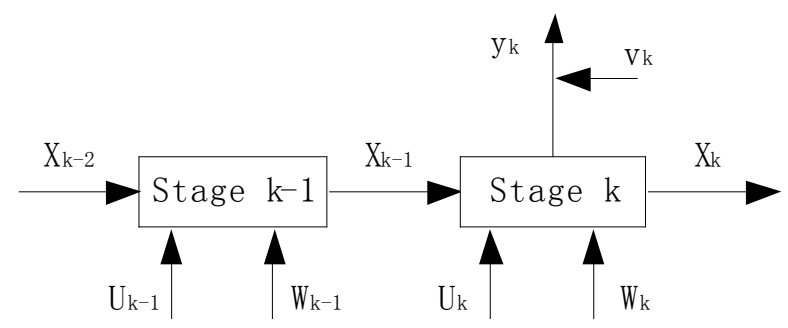

Fig. (1). Two sub processes tandem structure.

First of all, the two sub processes tandem structure is taken as an example to discuss the modeling of multistage manufacturing process variation transmission model, as shown in Fig. (1). On the basis of the AR (1) model propos Ced by Lawless (1999), the quality relationship of the adjacent two tandem structure sub processes in figure is formulized:

$$
\begin{aligned}
& x_{k}=A_{k-1} x_{k-1}+B_{k} u_{k}+W_{k} \\
& y_{k}=C_{k} x_{k}+v_{k}
\end{aligned}
$$

Where $A_{k-1} x_{k-1}$ is the influence of the product quality characteristic on stage product quality, which is propagated from $k-1$ stage. is the influence of stage product quality characteristics on product quality, which is caused by this stage process failure. $C_{k}$ maps the product quality status in the $k_{t h}$ process to the product quality measurement value, namely measure coefficient vector. To facilitate the understand, the measure coefficient vector is assumed to be the unit matrix and there is no measurement error in this paper. So the formula (6) can be simplified:

$$
x_{k}=A_{k-1} x_{k-1}+B_{k} u_{k}+W_{k}
$$

Before modeling variation propagation model, assume that the quality characteristics $x_{k}$ and $\mathrm{x}_{\mathrm{k}-1}$ in the k stage and the $\mathrm{k}-1$ stage obey the normal distribution:

$$
x_{k-1} \sim N\left(\mu_{k-1}, \sigma_{k-1}^{2}\right), x_{k} \sim N\left(\mu_{k}, \sigma_{k}^{2}\right)
$$

For convenience, defined $A_{k-1}=\beta_{k-1}, B_{k} u_{k}=\alpha_{k}$, substituting into the formula (7) to acquire:

$$
x_{k}=\alpha_{k}+\beta_{k-1} x_{k-1}+w_{k}
$$

In multistage manufacturing process, the quality variation in a sub process besides affected by this process, also in- cludes the variation influence propagated from the last process. So the quality variation $x_{k}$ can be formulized:

$$
\operatorname{Var}\left(x_{k}\right)=\operatorname{Var}\left(E\left(x_{k} \mid x_{k-1}\right)\right)+E\left(\operatorname{Var}\left(x_{k} \mid x_{k-1}\right)\right)
$$

Defining $E\left(x_{k}\right)=\mu_{k}, \operatorname{Var}\left(x_{k}\right)=\sigma_{k}^{2}$, the following formula can be got by formulas (8) and (9):

$$
\begin{aligned}
& \mu_{k}=\beta_{k-1} \mu_{k-1}+\alpha_{k} \\
& \sigma_{k}^{2}=\beta_{k-1}^{2} \sigma_{k-1}^{2}+\sigma_{k A}^{2}
\end{aligned}
$$

Formula (11) is the two stage process variation propagation model, the first item $\beta_{k-1}^{2} \sigma_{k-1}^{2}$ on the right side of the formula means the quality variation propagated from the $k-1$ stage to the $k$ stage. The second item $\sigma_{k A}^{2}$ said the increased quality variation of the $k$ stage itself in the manufacturing process, which include normal variation and the system noise.

Formulas (2) to (6) represents the two-stage quality characteristics variation propagation model, after recursion, the multistage manufacturing process variation propagation model can be got:

$$
\operatorname{Var}\left(x_{k}\right)=\sigma_{k}^{2}=\sum_{i=1}^{k} \beta_{k}^{2} \beta_{k-1}^{2}, \cdots, \beta_{i}^{2} \sigma_{i A}^{2}+\beta_{k}^{2} \beta_{k-1}^{2}, \cdots, \beta_{1}^{2} \sigma_{1 A}^{2}
$$

Formula (7) is the multistage manufacturing process variation propagation model.

\section{MULTISTAge MANUFACTURING PROCESS CA- PABILITY OPTIMIZATION}

In multistage manufacturing process, there would be different effects and influence of each sub processes quality variation on the total process capability and the final product improvement. Richard J. Linn et al. (2002) present the relationship between the each sub process quality variation and process capability indexes, and then determine the priorities of quality improvement. In this article, the interrelation between each sub process quality variation $\sigma^{2}$, transfer coefficient $\beta$ and $C_{p k}$ are respectively established based on the Linn's study. Then, the different effects of each sub process quality variation reduction on the specific process capability and overall process capability. At last, two concepts, "difficulty" and "cost-utility Ratio" are introduced to be as the improvement basis of multistage manufacturing process capability optimization.

\subsection{The Ideas of Multistage Manufacturing Process Ca- pability Optimization}

In this paper, The ideas of multistage manufacturing process capability optimization is keeping the other sub process quality variation invariable and improve the final process capability index by reducing one sub process quality variation. According to the above multistage manufacturing process modeling analysis. Two stage string manufacturing process variation propagation model is: 


$$
\begin{aligned}
& \mu_{2}=\alpha_{2}+\beta_{1} \mu_{1} \\
& \sigma_{o 2}^{2}=\beta^{2} \sigma_{o 1}^{2}+\sigma_{s 2}^{2}
\end{aligned}
$$

Among them, $\beta$ value can be obtained based on historical data using PLSR method. Therefore, for two stages manufacturing process, there are two ways to optimize the overall process capability:

Method 1: keep $\sigma_{s 2}=1$ unchanged, gradually reduce the first sub process quality variation.

Method 2: keep $\sigma_{s 1}=1$ unchanged, gradually reduce the second sub-process quality variation.

Detailed analysis of these two approaches as follows

According to the formula(12), $\sigma_{o 2}$ could get different values by different correlation coefficients $\beta$ and process capability index $C_{p k s i}(i=1,2)$. The relationship between $\sigma_{o 2}, C_{p k s i}, \beta$ and $\sigma_{s i}$ under two methods in Tables $\mathbf{1}$ and 2.

The relationship diagram between overall process capability $C_{p k o 2}$ and correlation coefficients $\beta$ could be got through the data of the Table $\mathbf{1}$ and 2, as shown in Figs. (2) and (3).

As seen in Fig. (2), according to the improved method 1 that improve the same degree of process capability index $C_{p k s 1}$, the bigger value of $\beta$, the greater increase of process capability index $C_{p k o 2}$, and if the value of $\mathrm{A}$ is fixed, then the distance between the curves is not the same. Therefore, it can be concluded that the improvement of process capability

Table 1. The relationship table of $\sigma_{o 2}, C_{p k s i}, \beta$ and $\sigma_{s i}$ in method 1.

\begin{tabular}{|cc|ccccccccc|}
\hline \multicolumn{10}{|c}{} & \multicolumn{10}{|c|}{$\boldsymbol{8}$} \\
\hline $\boldsymbol{C}_{\boldsymbol{p k s 1} \boldsymbol{1}}$ & $\hat{\boldsymbol{U}}_{\boldsymbol{s} \boldsymbol{1}}$ & $\mathbf{0 . 1}$ & $\mathbf{0 . 2}$ & $\mathbf{0 . 3}$ & $\mathbf{0 . 4}$ & $\mathbf{0 . 5}$ & $\mathbf{0 . 6}$ & $\mathbf{0 . 7}$ & $\mathbf{0 . 8}$ & $\mathbf{0 . 9}$ \\
\hline \hline 1.1 & 1.2121 & 1.007 & 1.029 & 1.064 & 1.111 & 1.169 & 1.236 & 1.311 & 1.393 & 1.48 \\
1.2 & 1.1111 & 1.006 & 1.024 & 1.054 & 1.094 & 1.144 & 1.202 & 1.267 & 1.338 & 1.414 \\
1.3 & 1.0256 & 1.005 & 1.021 & 1.046 & 1.081 & 1.124 & 1.174 & 1.231 & 1.294 & 1.361 \\
1.4 & 0.9524 & 1.005 & 1.018 & 1.04 & 1.07 & 1.108 & 1.152 & 1.202 & 1.257 & 1.317 \\
1.5 & 0.8889 & 1.004 & 1.016 & 1.035 & 1.061 & 1.094 & 1.133 & 1.178 & 1.227 & 1.281 \\
1.6 & 0.8333 & 1.003 & 1.014 & 1.031 & 1.054 & 1.083 & 1.118 & 1.158 & 1.202 & 1.25 \\
1.7 & 0.7843 & 1.003 & 1.012 & 1.027 & 1.048 & 1.074 & 1.105 & 1.141 & 1.181 & 1.224 \\
1.8 & 0.7407 & 1.003 & 1.011 & 1.024 & 1.043 & 1.066 & 1.094 & 1.126 & 1.162 & 1.202 \\
1.9 & 0.7018 & 1.003 & 1.01 & 1.022 & 1.039 & 1.06 & 1.085 & 1.114 & 1.147 & 1.183 \\
2.0 & 0.6667 & 1.002 & 1.009 & 1.02 & 1.035 & 1.054 & 1.077 & 1.104 & 1.133 & 1.166 \\
\hline
\end{tabular}

Table 2. The relationship table of $\sigma_{o 2}, C_{p k s 2}, \boldsymbol{\beta}$ and $\sigma_{s 2}$ in method 2 .

\begin{tabular}{|c|c|c|c|c|c|c|c|c|c|c|}
\hline \multicolumn{10}{|c|}{$\beta$} \\
\hline $\boldsymbol{C}_{p k s 2}$ & $\hat{\boldsymbol{U}}_{s 2}$ & 0.1 & 0.2 & 0.3 & 0.4 & 0.5 & 0.6 & 0.7 & 0.8 & 0.9 \\
\hline \hline 1.1 & 1.2121 & 1.216 & 1.228 & 1.249 & 1.276 & 1.311 & 1.352 & 1.4 & 1.452 & 1.51 \\
\hline 1.2 & 1.1111 & 1.116 & 1.129 & 1.151 & 1.181 & 1.218 & 1.263 & 1.313 & 1.369 & 1.43 \\
\hline 1.3 & 1.0256 & 1.03 & 1.045 & 1.069 & 1.101 & 1.141 & 1.188 & 1.242 & 1.301 & 1.364 \\
\hline 1.4 & 0.9524 & 0.958 & 0.973 & 0.999 & 1.033 & 1.076 & 1.126 & 1.182 & 1.244 & 1.31 \\
\hline 1.5 & 0.8889 & 0.895 & 0.911 & 0.938 & 0.975 & 1.02 & 1.072 & 1.131 & 1.196 & 1.265 \\
\hline 1.6 & 0.8333 & 0.839 & 0.857 & 0.886 & 0.924 & 0.972 & 1.027 & 1.088 & 1.155 & 1.227 \\
\hline 1.7 & 0.7843 & 0.791 & 0.809 & 0.84 & 0.88 & 0.93 & 0.987 & 1.051 & 1.12 & 1.194 \\
\hline 1.8 & 0.7407 & 0.747 & 0.767 & 0.799 & 0.842 & 0.894 & 0.953 & 1.019 & 1 & 1.166 \\
\hline 1.9 & 0.7018 & 0.709 & 0.73 & 0.763 & 0.808 & 0.862 & 0.923 & 0.991 & 1.064 & 1.141 \\
\hline 2.0 & 0.6667 & 0.674 & 0.696 & 0.731 & 0.777 & 0.833 & 0.897 & 0.967 & 1.041 & 1.12 \\
\hline
\end{tabular}




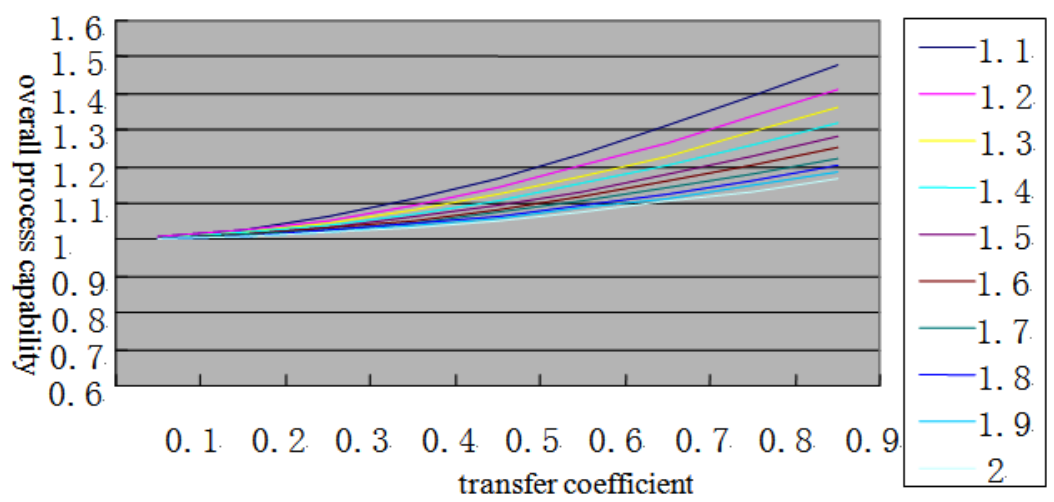

Fig. (2). The diagram of overall process capability and transfer coefficient in method 1 .

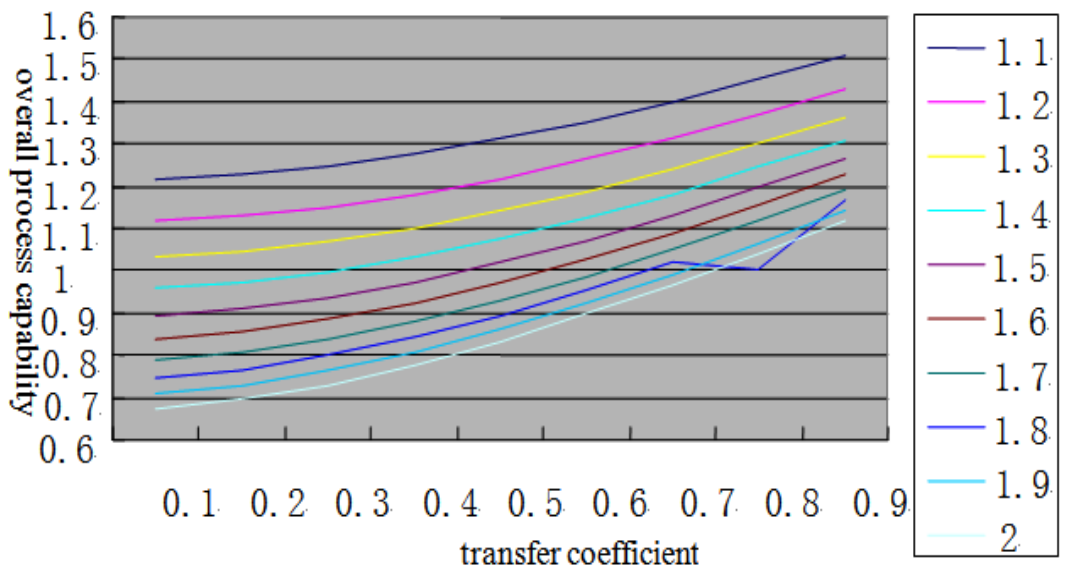

Fig. (3). The diagram of overall process capability and transfer coefficient in method 2.

index $C_{p k o 2}$ and the improvement of process capability index $C_{p k s 1}$ is not proportional if use method 1.If the initial value of $C_{p k s 1}$ is relatively high, $C_{p k s 1}$ need to be higher to increase the $C_{p k o 2}$ with same extent.

In Fig. (3), increasing the same amount process capability index $C_{p k s 2}$, the larger value of $\beta$, the smaller increase of process capability index $C_{p k o 2}$, if the value of $\beta$ is fixed, the distance between the two process capability curve are equal. Therefore, it can be concluded that the improvement of process capability index $C_{p k o 2}$ and the improvement of process capability index $C_{p k s 2}$ is proportional if use method 2. Because of the higher effect of sub-process, increasing the value of $\beta$ will increase the second sub fluctuations. Therefore, raising the same amount of B need greater efforts.

\subsection{Method of Multistage Manufacturing Process Capa- bility Optimization based on "Cost-Utility Ratio"}

The ultimate goal of multistage process capability optimization is clearly pointing out the relationship between different sub processes quality output and the process capability of the final output, enhancing the improvement effect of multistage manufacturing process capability. Therefore, based on the above analysis, multistage tandem process capability optimization method can be summarized as follows:

The first step: Through the historical data, the mean, variance and the transfer coefficient $\beta$ of each process need to be calculated based on the quality relationship model and variation transmission model.

Step two: calculating the specific and overall process capability index of each sub process according to the formulas (1), (2), (3) and (4);

Step Three: Compare $C_{p k o 2}$ and its target value, if it meet the target value, it do not need to be improved. Otherwise, the target value is set as improvement targets;

Step four: Based on the target of $C_{p k o 2}$ and the value of $\beta$, calculating the needed degree of quality variation reduction to achieve the target value of $C_{p k o 2}$ according to the two methods by the formula (12) and (13);

Step Five: According to the actual situation, calculate $F_{d i}$, then calculate cost-utility ratio $E R$ by the formul,

$$
E R=\frac{I C_{p k o k j}}{I C_{p k s i j} \times F_{d i j}} .
$$

According to the formula, the utility ratio is inversely proportional to the degree of difficulty in variation reduction, 
and proportional to the degree of improvement in overall process capacity. that is, the higher utility ratio indicating that the effect of improving process capacity by per unit of resource consumption is more excellent, so the best process ability optimized order could be determined.

\section{EMPIRICAL STUDY}

Now take mandrel drilling process as an example to verify the correctness and effectiveness of the method described above. This mandrel drilling process that mandrel after processing is carried fixture in the machine, then drilling by the tool, is a typical multi-stage manufacturing process, shown in Fig. (4).

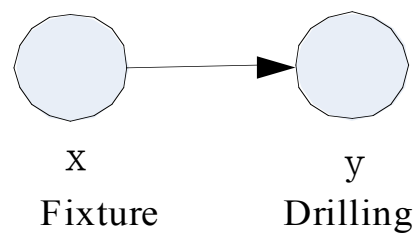

Fig. (4). Drilling mandrel manufacturing process diagram.
Among this, the key quality characteristics, fixture diameter, in the fixture process is $x, y$ for the hole diameter, which is the drilling process key quality characteristics, fixture diameter has a direct impact on the hole diameter after drilling.

Within a certain time, random sample of 70 sets data are chosen and ensure that they are one to one, specific data could be shown in Tables $\mathbf{3}$ and $\mathbf{4}$. Where, Table $\mathbf{3}$ is fixture diameter sample data and Table $\mathbf{4}$ is the hole diameter sample data.

Through the data statistical analysis, we can obtained that, during fixture process, the mean of fixture diameter $x$ is 201.24, variance is 2.618524. In the drilling process, the mean of hole diameter $y$ is 201.131, variance is 1.685 . Specification limit of fixture diameter is set as [202.38, 218.1], specification limit of hole diameter is set as [194.91, 207.35]. Assuming that the random errors of the two process were $\varepsilon_{x}$ and $\varepsilon_{y}$, and subject to normal distribution $\varepsilon_{x} \sim N\left(0, \sigma_{x}^{2}\right), \varepsilon_{y} \sim N\left(0, \sigma_{y}^{2}\right)$, in accordance with the foregoing modeling method, the quality relationship mode could be got:

Table 3. Fixture diameter sample data (unit: $0.001 \mathrm{~mm}$ ).

\begin{tabular}{|l|l|l|l|l|l|l|}
\hline 202.0256 & 202.8422 & 200.3642 & 199.5749 & 202.9619 & 201.2187 & 199.7900 \\
\hline 201.2319 & 201.5942 & 199.0821 & 201.6334 & 200.8429 & 200.3010 & 201.4587 \\
\hline 200.7930 & 201.6634 & 202.9758 & 199.2066 & 198.7843 & 204.6969 & 201.0150 \\
\hline 203.3055 & 203.2036 & 200.0877 & 200.6782 & 201.2558 & 200.8231 & 199.3574 \\
\hline 204.2553 & 200.7955 & 201.2217 & 199.7167 & 201.3555 & 198.9591 & 203.1555 \\
\hline 200.3944 & 201.0246 & 201.2387 & 199.3393 & 201.7522 & 204.1044 & 201.2150 \\
\hline 201.4074 & 199.1841 & 200.8364 & 199.5876 & 202.0488 & 201.7668 & 202.1077 \\
\hline 199.9331 & 198.5480 & 201.8817 & 200.5900 & 203.3082 & 199.4292 & 200.0807 \\
\hline 202.3411 & 200.1015 & 200.8128 & 201.5210 & 200.3535 & 202.2438 & 200.1792 \\
\hline 197.4137 & 201.6945 & 198.5473 & 201.0521 & 201.6620 & 203.2947 & 201.7487 \\
\hline
\end{tabular}

Table 4. Hole diameter sample data (unit: $0.001 \mathrm{~mm}$ ).

\begin{tabular}{|l|l|l|l|l|l|l|}
\hline 201.2696 & 202.2174 & 202.1843 & 200.2812 & 201.7033 & 199.8574 & 203.3839 \\
\hline 203.5300 & 200.1935 & 201.9570 & 199.7283 & 202.7937 & 200.2373 & 203.6458 \\
\hline 200.7739 & 200.1945 & 202.8315 & 201.0690 & 200.4849 & 202.8696 & 203.2533 \\
\hline 204.0029 & 200.8699 & 201.5556 & 201.6234 & 199.6789 & 199.9508 & 199.5008 \\
\hline 203.0891 & 201.1044 & 200.2575 & 200.7022 & 202.1793 & 200.5951 & 200.8538 \\
\hline 198.6063 & 201.4930 & 200.9372 & 200.4821 & 201.1845 & 200.4740 & 200.8728 \\
\hline 198.9497 & 202.5047 & 197.9522 & 201.0843 & 200.1494 & 203.2334 & 201.5301 \\
\hline 200.3866 & 201.9379 & 201.7453 & 200.9042 & 201.0153 & 201.2360 & 200.3881 \\
\hline 200.8898 & 198.8587 & 201.2828 & 199.8885 & 198.5235 & 199.7278 & 199.8620 \\
\hline 201.1426 & 202.0362 & 200.3637 & 202.8087 & 202.5379 & 199.6714 & 200.5510 \\
\hline
\end{tabular}


Table 5. Variation and process capability of two stage process.

\begin{tabular}{|ccc|}
\hline & $i=1$ & $i=2$ \\
\hline \hline$\sigma_{s i}$ & 2.618524 & 1.685 \\
$C_{p k s i}$ & 1 & 1.23 \\
$\sigma_{o i}$ & 2.618524 & 2.072036 \\
$C_{p k o i}$ & 1 & 1 \\
\hline
\end{tabular}

Table 6. Process capability analysis.

\begin{tabular}{|cccc|}
\hline & $\begin{array}{c}\text { Current } \\
\text { value }\end{array}$ & Method 1 & Method 2 \\
\hline \hline$\sigma_{s 1}$ & 2.618524 & $1.835(\downarrow 29.9 \%)$ & 2.618524 \\
$\sigma_{s 2}$ & 1.685 & 1.685 & $1.449(\downarrow 14 \%)$ \\
$\sigma_{o 2}$ & 2.072036 & $1.885(\downarrow 9 \%)$ & $1.885(\downarrow 9 \%)$ \\
& 1 & $1.428(\uparrow 42.8 \%)$ & 1 \\
$C_{p k o 2}$ & 1.23 & 1.23 & $1.431(\uparrow 16.3 \%)$ \\
$E R$ & 1 & $1.1(\uparrow 10 \%)$ & $1.1(\uparrow 10 \%)$ \\
& & 0.2336 & 0.4975 \\
\hline
\end{tabular}

$x=201.24+\varepsilon_{x} \quad y=104.325+0.460456 x+\varepsilon_{y}$

Because $\sigma_{s 1}=\sigma_{o 1}, C_{p k s 1}=C_{p k o 1}$, according to the formula (1) and (2), the specific process capability and overall process capability could be calculated, the results are shown in Table 5.

From the table, this two stage manufacturing process capability is obviously insufficient, the current process capability index $C_{p k o 2}$ is 1 , for ease to analysis, set making increased by $10 \%$ as an improve target, means when $C_{p k o 2}=1.1$, this process capability is acceptable.

Then using the above process capability analysis to reduce the quality variation of the two stage sub processes to improve process capability, the results are shown in Table 6.

As shown in Table 6, according to the formula (12), $\sigma_{s 1}$, $\sigma_{s 2}$ and $\sigma_{o 2}$ is $2.618524,1.685$ and 2.072036 respectively. The current $C_{p k o 2}$ is 1 , In order to achieve the goal of $C_{p k o 2}=1.1$.In the method 1 , reducing the fixture diameter quality variation $\sigma_{s 1}$ to 1.835 , correspondingly the $C_{p k s 1}$ increased to 1.428 , total process quality variation reduced to 1.885 , and achieve the target of $C_{p k o 2}=1.1$. Also in the method 2 , reducing the drilling process quality variation $\sigma_{s 2}$ to 1.449 , correspondingly the $C_{p k s 2}$ increased 16.3 percent to1.431, also achieved the target of $C_{p k o 2}=1.1$.
Two methods both achieve the goals of improving process capability, and therefore need to compare the cost-utility ratio in improving the process ability. Assumed the degree of difficulty $F_{d 1}=F_{d 2}=1$, then according to the formula (5), the cost-utility ratio is 0.2336 in method 1, and 0.4975 in method 2. Obviously, you should choose method 2 as the priority measures to improve the process capability.

\section{CONCLUSION}

In this paper, according to modeling analysis of multistage manufacturing process, the corresponding relationship between the sub processes quality variation, each sub process capability, the sub process transfer coefficient and overall process capability could be built, and identifying the influence of each sub processes quality variation and different transfer coefficient on overall process capability. And the "difficulty" and "cost-utility ratio" are introduced into evaluating the effect of quality improvement, which could provide the basis for the process ability optimization.

There are some limitations of this paper: (1) Sensitivity analysis problem of the multistage manufacturing process capability; (2) Multistage manufacturing process modeling and process capability analysis problems under parallel structure; (3) the multistage process ability analysis considering the mean shift. All this will be the focus of author's future work. 


\section{CONFLICT OF INTEREST}

The author confirms that this article content has no conflict of interest.

\section{ACKNOWLEDGEMENTS}

This work was supported by the National Natural Science Foundation of China (Grant No.71272207), the Ministry of Education of Humanities and Social Science Foundation of China(Grant No.14YJC630126)and Science and technology research key project Foundation in Henan province department of education of China (No. 14A630053).

\section{REFERENCES}

[1] S. Kotz and N. L. Johnson, "Process capability indices-a review, 1992-2000/discussion”, Journal of Quality Technology, vol. 34, no. 1, pp. $2-53,2002$.

[2] L. K. Chan, and S. W. Cheng, "Spring F A. A new measure of process capability index Cpm", Journal of Quality Technology, vol. 20 , no. 3, pp. $162-175,1988$

[3] C. W. Wu, W. L. Pearn, and S. Kotz, "An overview of theory and practice on process capability indices for quality assurance", Int. J. Production Economics, vol. 117, pp. 338-359, 2009.

[4] V. E. Kane, "Process capability indices", Journal of Quality Technology, vol. 18, pp. 41-52, 2009.

[5] B. H. Gunter, "The use and abuse of Cpk (Part 1-4)", Quality Progress, vol. 22, no. 1, pp. 72-73, 1989.

[6] R. A. Boyles, "The Taguchi capability index", Journal of Quality Technology, vol. 23, no. 1, pp. 107-126, 1991.
[7] S. Sardar and Y. H. Lee, "A Quantitative Model of Supply Chain Innovativeness as a Disruption Management Strategy in the Textile Industry", Advances in Industrial Engineering and Management, vol. 4, no. 1, pp. 9-28, 2015 .

[8] W. L. Pearn and C. S. Chang, "Precision measures for processes with multiple manufacturing lines", International Journal of $\mathrm{Ad}$ vanced Manufacturing Technology, no. 30, pp. 1202-1210, 2006.

[9] Y. Ding, S. Zhou, and Y. Chen, "A comparison of process variation estimators for in-process dimensional measurements and control", ASME Transactions, Journal of Dynamic Systems, Measurement and Control, vol. 127, no. 1, pp. 69-79, 2005.

[10] R. J. Linn, E. Au, and F. Tsung, "Process Capability Improvement for Multistage Processes", Quality Engineerig, vol. 15, no. 2, pp. 281-292, 2002.

[11] N. Wang, "Identifying Method for Key Quality Characteristics in Series-Parallel Multistage Manufacturing Process", The Open Automation and Control Systems Journal, vol. 11, no. 6, pp. 203207, 2014

[12] N. Wang, "Identifying method of key quality characteristics in multistage manufacturing process", Computer Integrated Manufacturing Systems, no. 4, pp. 888-895, 2013.

[13] N. Wang, "Method of Identifying Key Quality Characteristics in Multistage Manufacturing Process Based on PLSR", Operations Research and Management Science, no. 5, pp. 227-232, 2013.

[14] J. Sun, S. X. Wang, and Z. H. Fu, "Process capability analysis and estimation scheme for auto correlated data", Journal of Systems Science and Systems Engineering, vol. 19, no. 1, pp. 105$127,2010$.

[15] W. Z. Huang and Z. Y. Kong, "Process Capability Sensitivity Analysis for Design Evaluation of Multistage Assembly Processes", IEEE Transactions on Automation Science and Engineering, vol. 10, no. 7, pp. 736-745, 2010.

Received: September 16, 2014

Revised: December 23, 2014

Accepted: December 31, 2014

(C) Ning Wang; Licensee Bentham Open.

This is an open access article licensed under the terms of the (https://creativecommons.org/licenses/by/4.0/legalcode), which permits unrestricted, non-commercial use, distribution and reproduction in any medium, provided the work is properly cited. 